\title{
Visual outcomes in diabetic macular edema patients after avastin injection
}

\begin{abstract}
Objective: To determine the visual outcome in Diabetic Macular Edema patients after 3rd Avastin injections attending a tertiary eye care hospital.

Materials and methods: This was a cross sectional study with Non probability convenient sampling technique. The study was carried out at Diabetic clinic of Al-Ibrahim Eye Hospital, Isra Postgraduate Institute of Ophthalmology, Karachi-Pakistan. Ethical approval was taken from the institutional review board of Institute. Data collection were done retrospectively from January 2017 to June 2019. Data were retrieved for DME patients who have completed three follow-ups with Avastin injection. Inclusion Criteria were patients with age 30 to 60 years, Patient with PDR and NPDR with diabetic macular edema after $3^{\text {rd }}$ injection. Data Analysis was done using SPSS version 23.0.
\end{abstract}

Results: A total of 40 eyes of 40 patients were included in this study after getting information from the record sheet. Analysis were done in 30 eyes of 30 patients because 10 patients were missed their follow-up due to certain reason which were observed from record sheet. Mean age of patients was found to be $41.25 \pm 10.24$.Pre-operative Avastin injection best corrected visual acuity (BCVA) was noticed by using Log MAR without glasses was 0.49 and with glasses was 0.40 . Post-operative best corrected visual acuity Log MAR without glasses 0.51 and with glasses 0.42 after Avastin injection. Improvement of visual acuity was classified as Improved, worsen and Stable. There were 22 (73.33\%) patients observed with improvement in visual acuity, $5(16.66 \%)$ patients retained their vision stable and only 3 $(10 \%)$ patients worsen their visual acuity after all three Avastin injections.

Conclusion: The most common cause of diabetic macular edema is non-proliferative diabetic retinopathy and proliferative Diabetic Retinopathy. The Intravitreal injection play vital role, the timely treatment would improve prognosis of visual outcomes in Diabetic macular edema. So the study significantly shows the improvement in best corrected visual acuity before and after three visits.

Keywords: diabetic retinopathy, diabetic macular edema, visual acuity, avastin injection
Volume 9 Issue 6 - 2020

\author{
Charles Masih,' Kanwal Parveen, ${ }^{2}$ Samreen \\ Brohi, ${ }^{3}$ Shehar Bano Siyal, ${ }^{3}$ Fatima Zia, ${ }^{3}$ \\ Shabnam Pari Bhutto, ${ }^{3}$ Muhammad Faisal \\ Fahim $^{3}$ \\ 'Sindh Institute of Ophthalmology and Visual Sciences (SIOVS) \\ Hyderabad, Pakistan \\ 2Isra School of Optometry,Al-lbrahim Eye Hospital, Isra \\ Postgraduate Institute of Ophthalmology, Karachi, Pakistan \\ ${ }^{3}$ Isra School of Optometry, Al-Ibrahim Eye Hospital, Isra \\ Postgraduate Institute of Ophthalmology, Karachi, Pakistan
}

Correspondence: Muhammad Faisal Fahim, M.Sc. (Statistics), Researcher, Al-Ibrahim Eye Hospital, Isra Postgraduate Institute of Ophthalmology, Karachi-Pakistan, Email faisalfahim88@hotmail.com

Received: October 06, 2020 | Published: November 23, 2020
Abbreviations: DME, diabetic macular edema; DAP, diabetic association of pakistan; ETDRS, early treatment diabetic retinopathy study; VEGF, vascular endothelial growth factor; BCVA, best corrected visual acuity

\section{Introduction}

In patients with diabetic macular edema Intravitreal Avastin injections plays vital role, resulting in improvement of visual acuity. Clinical trials are the evidence that vision in patients with diabetic macular edema may improve by the increasing the number of antivascular endothelial growth factor injections or use of treatments with longer durations of action. ${ }^{1}$ A systematic review done by Yau JW, et al. reported 6.8\%. prevalence of diabetic macular edema (DME) individuals with diabetes. ${ }^{2}$ Worldwide number of diabetics can be estimated that people with DR will grow from 126.6 million in 2011 to 191.0 million by 2030 , and the number of people with VTDR will increase from 37.3 million to 56.3 million, if no urgent action is taken. ${ }^{3}$ In 1994 and 1998 The first National Diabetes Survey of Pakistan (1st NDSP) was done by the Diabetic Association of Pakistan (DAP) in collaboration with WHO. ${ }^{4}$ and In Pakistan Basit A, et al. Second National diabetes survey was reported in which prevalence of diabetes was $26.3 \%$, of which $19.2 \%$ had known diabetes, and $7.1 \%$ were newly diagnosed people with diabetes. ${ }^{5}$ Prevalence of DR in Pakistan was $28.78 \%{ }^{6}$ In 1985 study in Early Treatment Diabetic
Retinopathy Study (ETDRS) was conducted. ${ }^{7}$ However, laser was not beneficial especially in macular edema laser. ${ }^{8}$ It was believed that the Most of the retinal damage that characterizes the disease is from breakdown of the inner blood retinal barrier mediated by numerous growth factors such as vascular endothelial growth factor (VEGF). ${ }^{9,10}$ In Phase II randomized trials Based on these facts anti-VEGF agents like Pegaptanib sodium and Ranibzumab have been evaluated for diabetic macular edema. ${ }^{11,12} \mathrm{~A}$ Drug widely used off-label for treatment of ocular diseases like retinal vein occlusion, neovascular age-related macular degeneration, DME, proliferative diabetic retinopathy, rubeosis irides, and retinopathy of prematurity is Bevacizumab (Avastin, Genentech Inc., San Francisco, CA) it is a complete fulllength humanized antibody, it binds to and competitively inhibits all isoforms of the VEGF-A family. While bevacizumab is presently FDA approved for the treatment of metastatic colorectal cancer, metastatic breast cancer, and non-small cell lung cancer, ${ }^{13}$ This study was designed to determine the visual outcome in Diabetic Macular Edema (DME) patients after 3rd Avastin injection.

\section{Methodology}

In this study Non probability convenient sampling technique was used with study design of cross-sectional study. The study was carried out at Diabetic clinic of Al-Ibrahim Eye Hospital, Isra Postgraduate Institute of Ophthalmology, Karachi-Pakistan. Ethical approval was 
taken from the institutional review board of Institute. Data collection were done retrospectively from January 2017 to June 2019. Data were retrieved for DME patients who have completed three follow-ups with Avastin injection. Inclusion Criteria were patients with age 30 to 60 years, Patient with PDR and NPDR with diabetic macular edema after $3^{\text {rd }}$ injection. Exclusion Criteria were incomplete records, cataract, Patient with any corneal disease, opacity, Glaucoma and any other retinal disease. Data Collection Procedure includes best corrected visual acuity (BCVA), ocular examination findings of slit lamp and dilated fundus was examined with 90D (Volk) lens. FFA and retinal OCT was done using 3D OCT -2000 FA plus. BCVA was taken on each follow up visit till three visits. Statistical analysis was done from statistical package for social sciences (SPSS) version 23.0.All the continuous variables were presented as mean \pm SD and the entire categorical variable were presents as frequency and percentages. Bar and Pie charts were made to show the results. Paired sample t-test was applied to see the significance. $\mathrm{P}$-value $<0.05$ considered to be statistically significant.

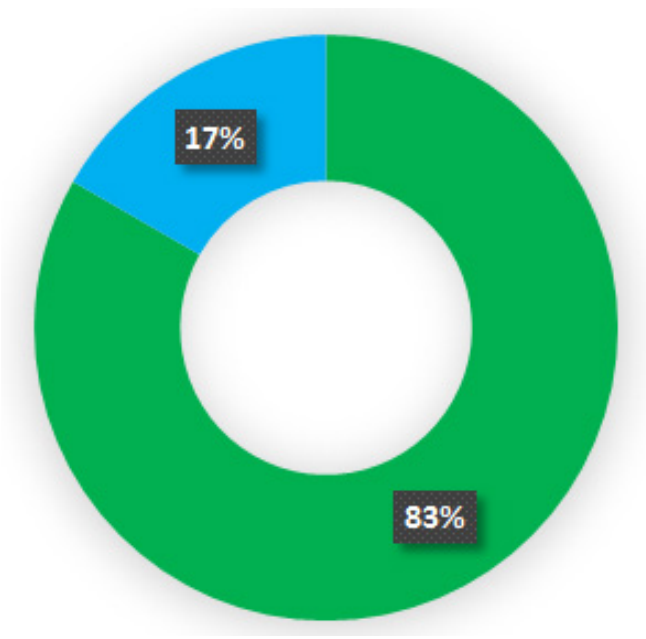

Figure I Status of retina.

Pre-operative Avastin injection best corrected visual acuity (BCVA) was noticed by using Log MAR without glasses was 0.49 and with glasses was 0.40 with significant P-value of 0.018 . Post-

\section{Results}

After getting information from the record sheet, A total of 40 eyes of 40 patients were included in this study Analysis were done in 30 eyes of 30 patients because 10 patients were missed their follow-up due to certain reason which were observed from record sheet. There were $53.3 \%$ right eye (OD) and 46.7\%left eye (OS). 41.25 \pm 10.24 years was found as Mean age of patient. Age groups were divided into three categories $4(13.4 \%)$ falling in age group $30-40$ years, $6(20.0 \%)$ were $41-50$ years and $20(66.7 \%)$ were in age group of 51-60 years. The ratio between Male to female was 1:1. Co-morbid was also asked from respondents $53.3 \%$ hypertensive and $46.7 \%$ non-Hypertensive. Mean Random blood sugar of all patients were $170.52 \pm 4.5$. Duration of diabetes were divided into three categories $<5$ years were $13.30 \%$, $5-10$ years were $10 \%$ and $>10$ years were $76.70 \%$. Tow type of Diabetic Retinopathy were observed NPDR+CSME+DME were $86.7 \%$ eyes and were $13.3 \%$ eyes. (Figure 1)

\section{- NPDR+CSME+DME \\ - PDR+CSME+DME}

operative best corrected visual acuity Log MAR without glasses 0.51 and with glasses 0.42 after Avastin injection with significant P-value of $<0.0001$. (Figure 2)

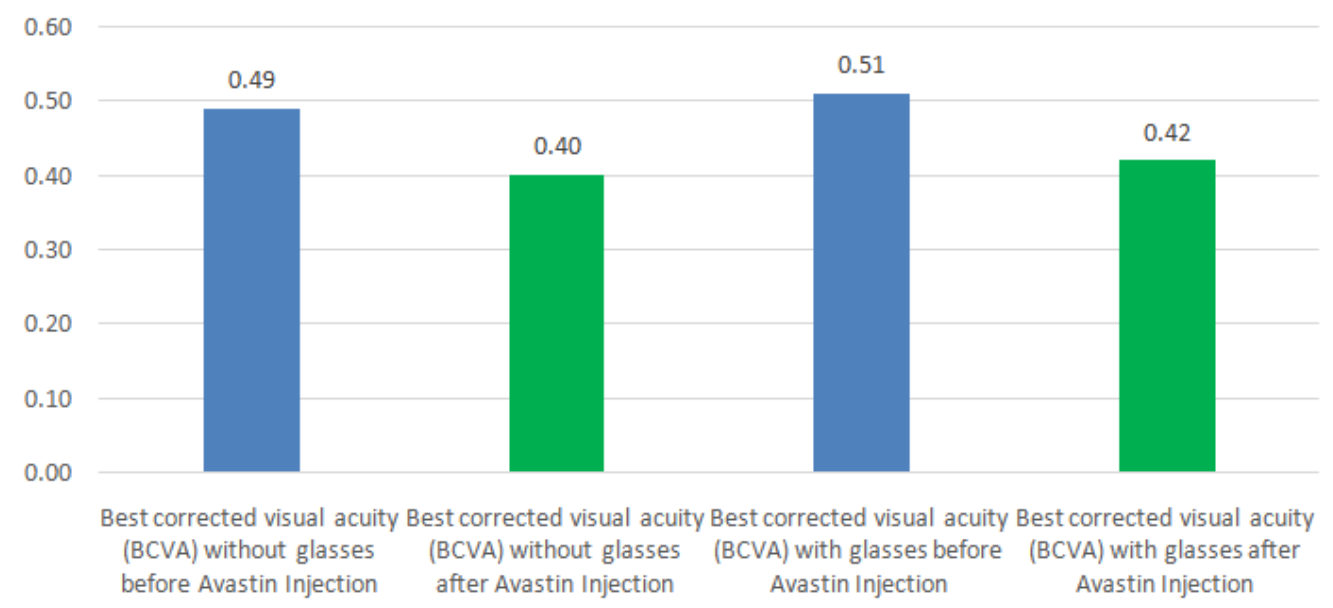

Figure 2 Mean best corrected visual acuity (logmar) before and after avastin injection.

*Preoperatively BCVA was significant at P-value of 0.018 .

*Post-operatively BCVA was significant at $P$-value $<0.001$.

*Paired sample t-test was applied to see the significance. 
Improvement of visual acuity was classified as Improved, worsen and Stable. There were $22(73.33 \%)$ patients observed with improvement in visual acuity, $5(16.66 \%)$ patients retained their vision stable and only $3(10 \%)$ patients worsen their visual acuity after all three Avastin injections. (Figure 3)

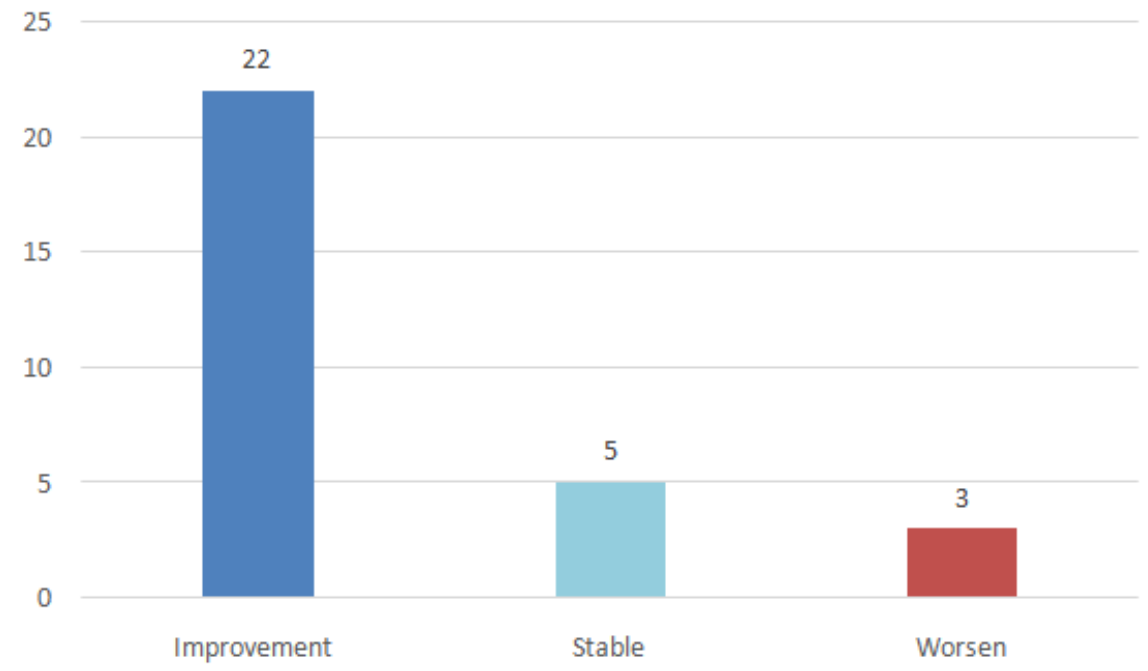

Figure 3 Visual acuity status after three avastin injection.

\section{Discussion}

This was an Observational cross-sectional study carried out at diabetic Clinic of Al Ibrahim Eye Hospital, Karachi. Purpose was to know best corrected visual acuity (BCVA) after three Avastin injection. In diabetic retinopathy most Common causes of visual loss are macular edema, vitreous haemorrhage and tractional retinal detachment. In patients of diabetic retinopathy, angiogenic mediators such as insulin like growth factor-1, erythropoietin, fibroblast growth factor and endothelial growth factor (VEGF) are released as a result of retinal ischemia and lead to the formation of new vessels in the retina. The result of neovascular growths Vitreous hemorrhage occurs and by precluding the retinal view, prevents panretinal photocoagulation, the gold standard treatment in proliferative diabetic retinopathy. Some of new opportunities for the treatment of retinal vascular disorder, the clinical use of anti-angiogenic agents has developed. it's a good choice for patients with vitreous hemorrhage Considering the anti angiogenic therapy, it accelerates the resolution of hemorrhage and facilitates PRP. ${ }^{14}$

In diabetic retinopathy, Diabetic macular edema is the main cause of decreased central vision. It can be diffuse or localized. Retinal thickening and hard exudates within $500 \mu \mathrm{m}$ of the center of the fovea, with associated atleast one disc diameter of retinal thickening (which may be outside the $500 \mu \mathrm{m}$ ) is seen in Clinically significant macular edema. ${ }^{15}$ Macular edema can be diagnosed clinical but we also confirm our diagnosis by fundus fluorescein angiography, the available investigation in our department. In resolving edema Antiangiogenic agents have been proved to be effective. The agent which we used was Avastin injection. In present study, improvement in BCVA were found in 22 eyes, no significant improvement / stable vision were observed in 5 eyes and visual acuity worsen in 3 eyes. Similar study done by Arevalo JF et al. ${ }^{16}$ stated that BCVA remained stable in 32 eyes and improved vision was seen in 43 eyes. A study by Jeong won seo et al. ${ }^{17}$ reported thirty eyes of twenty eight patients with DME included with the mean follow up period of 5.26+-2.39 months in which each patient completed 3 months follow up. In terms of BCVA remained same 15 eyes and 12 eyes were improved.

A Retrospective study done by Joshi L et al. There were 78 eyes from 54 patients. In $1^{\text {st }}$ course of Intravitreal Bevacizumab injection68\% eyes underwent succeeding cure from Diabetic macular edema. ${ }^{18}$ Some of studies reported that Intravitreal bevacizumab injection in patients with DME yielded a better visual outcome at 24 weeks compared with macular photocoagulation Soheilian M et al. ${ }^{19}$ An additional study Kook MD et al. reported that in cases with a longterm decrease of central retinal thickness in diffuse ischemic diabetic macular edema can be observed following repeated intravitreal injections of bevacizumab, also "treatment with bevacizumab at an earlier stage of diabetic macular edema without ischemia may be associated with an even better functional outcome". ${ }^{20}$

\section{Conclusion}

The most common cause of diabetic macular edema is nonproliferative diabetic retinopathy and proliferative Diabetic Retinopathy. The Intravitreal injection play vital role, the timely treatment would improve prognosis of visual outcomes in Diabetic macular edema. So the study significantly shows the improvement in best corrected visual acuity before and after three visits.

\section{Financial disclosure}

None.

\section{Conflicts of interest}

The authors declared that there are no conflicts between them.

\section{References}

1. Fong DS, Luong TQ, Contreras R, et al. Treatment patterns and 2-year vision outcomes with bevacizumab in diabetic macular edema: an analysis from a large us integrated health care system. Retina (Philadelphia, Pa.). 2018;38(9):1830-1838. 
2. Yau JW, Rogers SL, Kawasaki R, et al. Global prevalence and major risk factors of diabetic retinopathy. Diabetes Care. 2012;35(3):556-564.

3. Zheng Y, He M, Congdon N. The worldwide epidemic of diabetic retinopathy. Indian journal of ophthalmology. 2012;60(5):428-431.

4. Shera AS, Basit A, Fawwad A, et al. Pakistan National Diabetes Survey: prevalence of glucose intolerance and associated factors in the Punjab Province of Pakistan. Prim Care Diabetes. 2010;4(2):79-83.

5. Basit A, Fawwad A, Qureshi H, et al. Prevalence of diabetes, pre-diabetes and associated risk factors: second National Diabetes Survey of Pakistan (NDSP), 2016-2017. BMJ open. 2018;8(8):e020961.

6. Mumtaz SN, Fahim MF, Arslan M, et al. Prevalence of diabetic retinopathy in Pakistan: A systematic review. Pak J Med Sci. 2018;34(2):493-500.

7. Early Treatment Diabetic Retinopathy Study Research Group. Photocoagulation for diabetic macular edema: Early Treatment Diabetic Retinopathy Study report number 1. Arch Ophthalmol. 1985;103(12):17961806.

8. Diabetic Retinopathy Clinical Research Network. A randomized trial comparing intravitreal triamcinolone acetonide and focal $/$ grid photocoagulation for diabetic macular edema. Ophthalmology. 2008; 115(9):1447-1459.

9. Adamis AP, Miller JW, Bernal MT, et al. Increased vascular endothelial growth factor levels in the vitreous of eyes with proliferative diabetic retinopathy. Am J Ophthalmol. 1994;118(4):445-450.

10. Ishida S, Usui T, Yamashiro K. VEGF 164 is proinflammatory in the diabetic retina. Invest Ophthalmol Vis Sci. 2003;44:2155-2162.

11. Cunningham ET, Adamis AP, Altaweel M, et al. A phase II randomized double-masked trial of pegaptanib, an antivascular endothelial growth factor aptamer, for diabetic macular edema. Ophthalmology. 2005;112(10):1747-1757.

12. Chun DW, Heier JS, Topping TM, et al. A pilot study of multiple intravitreal injections of ranibzumab in patients with center-involving clinically significant diabetic macular edema. Ophthalmology. 2006;113(10):17061712 .
13. Falavarjani KG, Nguyen QD. Adverse events and complications associated with intravitreal injection of antiVEGF agents: a review of literature. Eye(Lond). 2013;27(7):787-794.

14. Alagoz C, Yildirim Y, Kocamaz M, et al. The efficacy of intravitreal Bevacizumab in vitreous hemorrhage of diabetic subjects. Turk $J$ Ophthalmol. 2016; 46(5): 221-225.

15. Chun DW, Heier JS, Topping TM. A pilot study of multiple intravitreal injections of Ranibizumab in patients with center involving clinically significant macular edema. Ophthalmology. 2006;113:1706-1712.

16. Arevalo JF1, Fromow-Guerra J, Quiroz-Mercado H, et al. Pan-american collaborative retina study group 'primary intravitreal bevacizumab (avastin) for diabetic macular edema: results from the pan-american collaborative retina study group at 6-month follow-up.', Ophthalmology. 2007;114(4):743-750.

17. Jeong Won Seo, MD and In Won Park, MD (March 09, 2009) 'Intravitreal Bevacizumab for Treatment of Diabetic Macular Edema. Korean $J$ Ophthalmol. 23(1):17-22.

18. Lavish Joshi, Asaf Bar, Oren Tomkins-Netzer, et al. Intravitreal bevacizumab injections for diabetic macular edema - predictors of response: a retrospective study. Clin Ophthalmol. 2016;10(2016):20932098.

19. Soheilian M, Ramezani A, Obudi A, et al. Randomized trial of intravitreal bevacizumab alone or combined with triamcinolone versus macular photocoagulation in diabetic macular edema. Am Acad Ophthalmol (Elsevier Inc.). 2009;116(6):1143-1150.

20. Kook D, Wolf A, Kreutzer T, et al. Long-term effect of intravitreal bevacizumab (avastin) in patients with chronic diffuse diabetic macular edema. Retina. 2008;2(8):1053-1060. 